\title{
Accurate and Efficient Simulation of Multiple Uncorrelated Rayleigh Fading Waveforms
}

\author{
Cheng-Xiang Wang, Member, IEEE, Matthias Pätzold, Senior Member, IEEE, \\ and Dongfeng Yuan, Senior Member, IEEE
}

\begin{abstract}
Simulating wideband fading channels, multipleinput multiple-output (MIMO) channels, and diversity-combined fading channels often demands the generation of multiple uncorrelated Rayleigh fading waveforms. In this letter, two appropriate parameter computation methods, namely the method of exact Doppler spread (MEDS) and $L_{p}$-norm method (LPNM), for deterministic sum-of-sinusoids $(\mathrm{SoS})$ channel simulators are investigated to guarantee the uncorrelatedness between different simulated Rayleigh fading processes. Numerical and simulation results show that the resulting deterministic SoS channel simulator can accurately and efficiently reproduce all the desired statistical properties of the reference model.
\end{abstract}

Index Terms-Multipath fading channels, Rayleigh processes, sum-of-sinusoids channel simulator, statistics.

\section{INTRODUCTION}

$\mathbf{T}$ HE generation of multiple uncorrelated Rayleigh fading waveforms is often required for simulating wideband fading channels, multiple-input multiple-output (MIMO) channels, and diversity-combined fading channels. It is therefore of great significance to develop channel simulators capable of accurately and efficiently simulating multiple uncorrelated Rayleigh fading processes. Jakes' deterministic sum-ofsinusoids (SoS) channel simulator [1] has extensively been applied to the simulation of Rayleigh fading channels. In order to generate multiple uncorrelated Rayleigh fading signals, Jakes [1] and other researchers [2]-[4] have investigated different methods to parameterize the underlying deterministic SoS channel simulators. Although Jakes' simulator [1] and its derivatives [2]-[4] are of deterministic nature, which has the advantage of simulation efficiency, they still retain some undesirable properties. For example, the crosscorrelation function (CCF) of any pair of underlying complex processes is generally not zero for the models in [1]-[3], and the drawback of the model in [4] is that the inphase and quadrature components of each underlying complex process have different autocorrelation functions (ACFs). To remedy the drawbacks of the deterministic channel simulators in [1]-[4], Zheng and Xiao [5], [6] reintroduced random parameters into the employed sinusoids, resulting in non-ergodic stochastic

Manuscript received June 30, 2005; revised January 10, 2006; accepted March 1, 2006. The associate editor coordinating the review of this paper and approving it for publication was R. Murch.

C.-X. Wang is with the Joint Research Institute for Signal and Image Processing, Heriot-Watt University, Edinburgh, UK (e-mail: chengxiang.wang@hw.ac.uk).

M. Pätzold is with the Faculty of Engineering and Science, Agder University College, Grimstad, Norway (e-mail: matthias.paetzold@hia.no).

D. Yuan is with the School of Information Science \& Engineering, Shandong University, Jinan, P. R. China (e-mail: dfyuan@sdu.edu.cn).

Digital Object Identifier 10.1109/TWC.2007.05503.
SoS channel simulators. By averaging over a large number of simulation trials, the developed stochastic channel simulators in [5], [6] can approximate closely the desired statistical properties. However, relatively high computational complexity has to be paid for the channel simulators in [5], [6] due to their non-ergodic stochastic nature.

In this letter, we go back to the deterministic SoS channel modeling approach in order to keep the simulation efficiency. The accuracy of its statistical properties is achieved by carefully determining the simulation model parameters. Two parameter computation methods, the method of exact Doppler spread (MEDS) and $L_{p}$-norm method (LPNM) [7], are revisited and the additional boundary conditions are investigated for producing multiple uncorrelated Rayleigh fading waveforms. Our analysis reveals an inappropriate remark given in [7, p. 284] for the MEDS concerning how to choose the numbers of sinusoids in order to guarantee the complete uncorrelatedness between different simulated processes. The widely used statistical properties of the channel simulator are studied in terms of the envelope probability density function (PDF), the ACF and CCF of the quadrature components of one complex process, the ACF of the complex process, and the $\mathrm{CCF}$ of any pair of simulated complex processes. In addition, the ACF of the squared envelope is, for the first time, derived for the deterministic SoS channel simulator. This is a fourthorder statistical quantity useful for estimating mobile speeds in handoff schemes [6]. The numerical and simulation results highlight the advantages of the presented channel simulator over other forms of channel simulators in [1]-[6] in both accurate reproduction of all the desired statistical properties of the reference model and efficient implementation due to the retained deterministic nature.

\section{The RefEREnCE Model}

Our aim is to generate $\mathcal{L}$ uncorrelated Rayleigh fading processes. It is well-known that a Rayleigh process is formed by taking the absolute value of a zero-mean complex Gaussian random process. Ideally, these $\mathcal{L}$ uncorrelated complex Gaussian random processes should satisfy the following criteria: 1) The inphase and quadrature components of each complex process are zero-mean independent real Gaussian random processes with identical ACFs; 2) The CCF of any pair of complex Gaussian random processes must be zero.

Let us denote the desired $\ell$ th $(\ell=1,2, \ldots, \mathcal{L})$ Rayleigh fading process by $\zeta_{\ell}(t)$, which is given by

$$
\zeta_{\ell}(t)=\left|\mu_{\ell}(t)\right|=\left|\mu_{1, \ell}(t)+j \mu_{2, \ell}(t)\right| .
$$


Here, $j=\sqrt{-1}, \mu_{\ell}(t)$ is a zero-mean complex Gaussian random process, $\mu_{1, \ell}(t)$ and $\mu_{2, \ell}(t)$ are uncorrelated real Gaussian random processes with common variance $\sigma_{0}^{2}$. The envelope PDF of $\zeta_{\ell}(t)$ is the Rayleigh distribution [1]

$$
p_{\zeta_{\ell}}(x)=\frac{x}{\sigma_{0}^{2}} \exp \left(-\frac{x^{2}}{2 \sigma_{0}^{2}}\right), \quad x \geq 0 .
$$

Adopting Clark's two-dimensional isotropic scattering theory [1], [8], the statistical properties of the reference model are specified by the following ACFs and CCFs [9]:

$$
\begin{aligned}
r_{\mu_{i, \ell} \mu_{i, \ell}}(\tau) & =E\left\{\mu_{i, \ell}(t) \mu_{i, \ell}(t+\tau)\right\} \\
& =\sigma_{0}^{2} J_{0}\left(2 \pi f_{\max } \tau\right) \\
r_{\mu_{1, \ell} \mu_{2, \ell}}(\tau) & =E\left\{\mu_{1, \ell}(t) \mu_{2, \ell}(t+\tau)\right\}=0 \\
r_{\mu_{2, \ell} \mu_{1, \ell}}(\tau) & =E\left\{\mu_{2, \ell}(t) \mu_{1, \ell}(t+\tau)\right\}=0 \\
r_{\mu_{\ell} \mu_{\ell}}(\tau) & =E\left\{\mu_{\ell}^{*}(t) \mu_{\ell}(t+\tau)\right\} \\
& =2 \sigma_{0}^{2} J_{0}\left(2 \pi f_{\max } \tau\right) \\
r_{\mu_{\ell} \mu_{\lambda}}(\tau) & =E\left\{\mu_{\ell}^{*}(t) \mu_{\lambda}(t+\tau)\right\}=0 \\
r_{\zeta_{\ell}^{2} \zeta_{\ell}^{2}}(\tau) & =E\left\{\zeta_{\ell}^{2}(t) \zeta_{\ell}^{2}(t+\tau)\right\} \\
& =4 \sigma_{0}^{4}+4 \sigma_{0}^{4} J_{0}^{2}\left(2 \pi f_{\max } \tau\right)
\end{aligned}
$$

for $i=1,2$ and $\ell, \lambda=1,2, \ldots, \mathcal{L}$ with $\ell \neq \lambda$. Here, $E\{\cdot\}$ refers to the statistical average operator, $f_{\max }$ is the maximum Doppler frequency, and $J_{0}(\cdot)$ denotes the zeroth-order Bessel function of the first kind. The goal of our channel simulator is then to reproduce the above desired statistical properties as accurately and efficiently as possible.

\section{The Deterministic SoS Simulation Model}

The central limit theorem justifies that a Gaussian random process can be approximated by the superposition of a large number of properly weighted sinusoids. This fact actually serves as the foundation of SoS channel simulators. For our simulation model, the $\ell$ th $(\ell=1,2, \ldots, \mathcal{L})$ Rayleigh fading process is modeled as

$$
\tilde{\zeta}_{\ell}(t)=\left|\tilde{\mu}_{\ell}(t)\right|=\left|\tilde{\mu}_{1, \ell}(t)+j \tilde{\mu}_{2, \ell}(t)\right|
$$

where

$$
\tilde{\mu}_{i, \ell}(t)=\sum_{n=1}^{N_{i, \ell}} c_{i, n, \ell} \cos \left(2 \pi f_{i, n, \ell} t+\theta_{i, n, \ell}\right), \quad i=1,2 .
$$

Here, $N_{i, \ell}$ defines the number of sinusoids, mainly determining the realization expenditure and the accuracy of the resulting channel simulator. The gains $c_{i, n, \ell}$, the discrete frequencies $f_{i, n, \ell}$, and the phases $\theta_{i, n, \ell}$ are real-valued parameters, which are kept constant during simulation. Consequently, $\tilde{\mu}_{i, \ell}(t)$ is a deterministic function and the resulting channel simulator is of deterministic feature. It follows that the statistical properties of our deterministic SoS channel simulator must be calculated by using time averages instead of statistical averages. The envelope PDF $\tilde{p}_{\zeta_{\ell}}(x)$ of $\tilde{\zeta}_{\ell}(t)$ can be computed by [7]

$$
\tilde{p}_{\zeta_{\ell}}(x)=x \int_{0}^{2 \pi} \tilde{p}_{\mu_{1, \ell}}(x \cos \theta) \cdot \tilde{p}_{\mu_{2, \ell}}(x \sin \theta) d \theta
$$

where

$$
\begin{gathered}
\tilde{p}_{\mu_{i, \ell}}(x)=2 \int_{0}^{\infty}\left[\prod_{n=1}^{N_{i, \ell}} J_{0}\left(2 \pi c_{i, n, \ell} \nu\right)\right] \cos (2 \pi \nu x) d \nu \\
i=1,2
\end{gathered}
$$

The time-averaged correlation functions of the simulation model, corresponding to (3)-(8) of the reference model, can be expressed as follows:

$$
\begin{aligned}
& \tilde{r}_{\mu_{i, \ell} \mu_{i, \ell}}(\tau)=\sum_{n=1}^{N_{i, \ell}} \frac{c_{i, n, \ell}^{2}}{2} \cos \left(2 \pi f_{i, n, \ell} \tau\right) \\
& \tilde{r}_{\mu_{1, \ell} \mu_{2, \ell}}(\tau)= \begin{cases}\sum_{n=1}^{N_{1, \ell}} \sum_{m=1}^{N_{2, \ell}} \frac{c_{1, n, \ell} c_{2, m, \ell}}{2} \cos \left(2 \pi f_{1, n, \ell} \tau\right. \\
\left.-\theta_{1, n, \ell} \pm \theta_{2, m, \ell}\right), & \text { if } f_{1, n, \ell}= \pm f_{2, m, \ell} \\
0, & \text { if } f_{1, n, \ell} \neq \pm f_{2, m, \ell}\end{cases} \\
& \tilde{r}_{\mu_{2, \ell} \mu_{1, \ell}}(\tau)=\tilde{r}_{\mu_{1, \ell} \mu_{2, \ell}}(-\tau) \\
& \tilde{r}_{\mu_{\ell} \mu_{\ell}}(\tau)=\sum_{i=1}^{2} \tilde{r}_{\mu_{i, \ell} \mu_{i, \ell}}(\tau) \\
& +j\left[\tilde{r}_{\mu_{1, \ell} \mu_{2, \ell}}(\tau)-\tilde{r}_{\mu_{2, \ell} \mu_{1, \ell}}(\tau)\right] \\
& \tilde{r}_{\mu_{\ell} \mu_{\lambda}}(\tau)=\tilde{r}_{\mu_{1, \ell} \mu_{1, \lambda}}(\tau)+\tilde{r}_{\mu_{2, \ell} \mu_{2, \lambda}}(\tau) \\
& +j\left[\tilde{r}_{\mu_{1, \ell} \mu_{2, \lambda}}(\tau)-\tilde{r}_{\mu_{2, \ell} \mu_{1, \lambda}}(\tau)\right], \ell \neq \lambda \\
& \tilde{r}_{\zeta_{\ell}^{2} \zeta_{\ell}^{2}}(\tau)=\sum_{i=1}^{2}\left\{\left(\sum_{n=1}^{N_{i, \ell}} \frac{c_{i, n, \ell}^{2}}{2}\right)^{2}\right. \\
& +2\left[\sum_{n=1}^{N_{i, \ell}} \frac{c_{i, n, \ell}^{2}}{2} \cos \left(2 \pi f_{i, n, \ell} \tau\right)\right]^{2} \\
& \left.-\sum_{n=1}^{N_{i, \ell}} \frac{c_{i, n, \ell}^{4}}{8}\left[2+\cos \left(4 \pi f_{i, n, \ell} \tau\right)\right]\right\} \\
& +\frac{1}{2} \sum_{n=1}^{N_{1, \ell}} c_{1, n, \ell}^{2} \sum_{m=1}^{N_{2, \ell}} c_{2, m, \ell}^{2} .
\end{aligned}
$$

In (17), the CCFs $\tilde{r}_{\mu_{i, \ell} \mu_{k, \lambda}}(\tau)$ between $\tilde{\mu}_{i, \ell}(t)$ and $\tilde{\mu}_{k, \lambda}(t)$ $(i, k=1,2$ and $\ell, \lambda=1,2, \ldots, \mathcal{L}$ with $\ell \neq \lambda)$ are given by

$\tilde{r}_{\mu_{i, \ell} \mu_{k, \lambda}}(\tau)= \begin{cases}\sum_{n=1}^{N_{i, \ell}} \sum_{m=1}^{N_{k, \lambda}} \frac{c_{i, n, \ell} c_{k, m, \lambda}}{2} & \cos \left(2 \pi f_{i, n, \ell} \tau\right. \\ \left.-\theta_{i, n, \ell} \pm \theta_{k, m, \lambda}\right), & \text { if } f_{i, n, \ell}= \pm f_{k, m, \lambda} \\ 0, & \text { if } f_{i, n, \ell} \neq \pm f_{k, m, \lambda} .\end{cases}$

In the Appendix, we have provided a brief outline for the derivation of the squared envelope $\mathrm{ACF} \tilde{r}_{\zeta_{\ell}^{2} \zeta_{\ell}^{2}}(\tau)$ in (18). The derivations of (13)-(17) are rather simple and the details are omitted here for brevity.

From (14), (15), and (19), it is clear that different processes $\tilde{\mu}_{i, \ell}(t)$ and $\tilde{\mu}_{k, \lambda}(t)(i, k=1,2$ and $\ell, \lambda=1,2, \ldots, \mathcal{L} ; i=k$ and $\ell=\lambda$ do not hold at the same time) are uncorrelated if

$$
f_{i, n, \ell} \neq \pm f_{k, m, \lambda}
$$

holds for all $n=1,2, \ldots, N_{i, \ell}$ and $m=1,2, \ldots, N_{k, \lambda}$. This means that the discrete frequencies for different uncorrelated 
processes must be disjoint. The inequality (20) further allows us to write $\tilde{r}_{\mu_{1, \ell} \mu_{2, \ell}}(\tau)=r_{\mu_{1, \ell} \mu_{2, \ell}}(\tau)=0, \tilde{r}_{\mu_{2, \ell} \mu_{1, \ell}}(\tau)=$ $r_{\mu_{2, \ell} \mu_{1, \ell}}(\tau)=0$, and $\tilde{r}_{\mu_{\ell} \mu_{\lambda}}(\tau)=r_{\mu_{\ell} \mu_{\lambda}}(\tau)=0$. Subsequently, two parameter computation methods (MEDS and LPNM) will be revisited concerning how to fulfill the desired boundary constraint (20).

\section{A. MEDS}

With the MEDS [7], the phases $\theta_{i, n, \ell}$ in (10) are simply considered as the outcomes of a random generator uniformly distributed over $(0,2 \pi]$, while $c_{i, n, \ell}$ and $f_{i, n, \ell}$ are given by

$$
\begin{aligned}
c_{i, n, \ell} & =\sigma_{0} \sqrt{\frac{2}{N_{i, \ell}}} \\
f_{i, n, \ell} & =f_{\max } \sin \left(\Phi_{i, n, \ell}\right)
\end{aligned}
$$

respectively, where

$$
\Phi_{i, n, \ell}=\frac{(2 n-1) \pi}{4 N_{i, \ell}} .
$$

We can easily show that the deterministic process $\tilde{\mu}_{i, \ell}(t)$ in (10) with the above chosen parameters has the desired mean value 0 and variance $\sigma_{0}^{2}$. It follows from $1 \leq n \leq N_{i, \ell}$ that $\frac{\pi}{4 N_{i, \ell}} \leq \Phi_{i, n, \ell} \leq \frac{\left(2 N_{i, \ell}-1\right) \pi}{4 N_{i, \ell}}$ holds. We may further write $0<\Phi_{i, n, \ell}<\pi / 2$ if $N_{i, \ell}<\infty$. Within this range, Equation (22) clearly indicates that the discrete frequencies $f_{i, n, \ell}$ are monotonously increasing values over the interval $\left(0, f_{\max }\right)$ with the increase of $n$, i.e., $0<f_{i, n, \ell}<f_{i, n+1, \ell}<f_{\max }$. As a result, $f_{i, n, \ell} \neq-f_{k, m, \lambda}$ can always be satisfied. The substitution of (22) into (20) tells us that $f_{i, n, \ell} \neq f_{k, m, \lambda}$ is guaranteed if and only if $\Phi_{i, n, \ell} \neq \Phi_{k, m, \lambda}$ holds, which results in

$$
\frac{N_{i, \ell}}{N_{k, \lambda}} \neq \frac{2 n-1}{2 m-1}
$$

for $n=1,2, \ldots, N_{i, \ell}, m=1,2, \ldots, N_{k, \lambda}, i, k=1,2$, and $\ell, \lambda=1,2, \ldots, \mathcal{L}(i=k$ and $\ell=\lambda$ do not hold at the same time). It is important to stress here that the expression (24) points out a non-sufficient statement in [7, p. 284], where it is claimed that the condition $N_{i, \ell} \neq N_{k, \lambda}$ is sufficient to ensure $f_{i, n, \ell} \neq f_{k, m, \lambda}$. From (24), we conclude that the ratio of $N_{i, \ell}$ to $N_{k, \lambda}$ must be unequal to the ratio of two odd numbers. Therefore, at maximum one odd value is allowed for all $N_{i, \ell}$ $(i=1,2$ and $\ell=1,2, \ldots, \mathcal{L})$. To simulate $\mathcal{L}=4$ uncorrelated Rayleigh fading channels by using the MEDS, an example set of 8 values for the numbers of sinusoids $N_{i, \ell}$ fulfilling (14) is $\{8,9,10,12,16,32,64,128\}$.

An obvious disadvantage with the above selection is that very large values have to be chosen for $N_{i, \ell}$, which increases greatly the complexity of our channel simulator, when $\mathcal{L}>4$ uncorrelated Rayleigh processes are simulated. Fortunately, this problem can be mitigated by the following two steps. First, let us choose the numbers of sinusoids $N_{i, \ell}$ in such a way that (24) is not fulfilled for only few pairs of $(n, m)$. In this case, the CCFs of any pair of processes are so small that they can be neglected in practice. Also, large values of $N_{i, \ell}$ can in principle be avoided. Substituting (21) into (19), it is clear that the maximum value of the CCF is $\tilde{r}_{\mu_{i, \ell} \mu_{k, \lambda}}^{\max }=\sum_{n=1}^{N_{i, \ell}} \sum_{m=1}^{N_{k, \lambda}} \frac{c_{i, n, \ell} c_{k, m, \lambda}}{2}=$

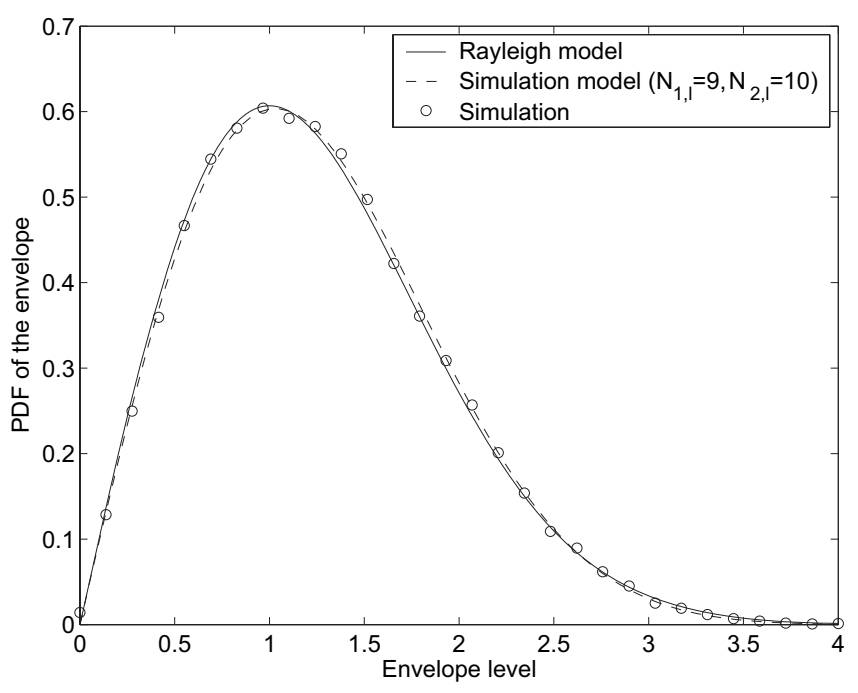

Fig. 1. The envelope PDFs of the Rayleigh model and the simulation model by using the $\operatorname{MEDS}\left(\sigma_{0}^{2}=1\right)$.

$\sigma_{0}^{2} P_{\mu_{i, \ell} \mu_{k, \lambda}}^{e} / \sqrt{N_{i, \ell} N_{k, \lambda}}$, where $P_{\mu_{i, \ell} \mu_{k, \lambda}}^{e}$ defines the number of pairs of $(n, m)$ fulfilling the equality $f_{i, n, \ell}=f_{k, m, \lambda}$. For example, to simulate a 12-path $(\mathcal{L}=12)$ wideband channel, a possible set of 24 values for $N_{i, \ell}$ is $\{8,9,11,13,16,17,18,19,22,23,25,26,28,29,31,32,34,36$, $37,41,43,47,51,53\}$. Our investigations show that the maximum cross-correlation between $\tilde{\mu}_{i, \ell}(t)$ and $\tilde{\mu}_{k, \lambda}(t)$ occurs when $N_{i, \ell}=18$ and $N_{k, \lambda}=22$. Then, $f_{i, n, \ell}=f_{k, m, \lambda}$ holds for $P_{\mu_{i, \ell} \mu_{k, \lambda}}^{e}=2$ pairs of $(n, m):(5,6)$ and $(14,17)$. As a result, $\widetilde{r}_{\mu_{i, \ell} \mu_{k, \lambda}}^{\max }=2 / \sqrt{18 \times 22} \approx 0.1005$ with $\sigma_{0}^{2}=1$. The second step is to further replace $f_{i, n, \ell}$ by $f_{i, n, \ell}+\varepsilon$ when $f_{i, n, \ell}=f_{k, m, \lambda}$ holds. Here, $\varepsilon$ is an infinitesimal quantity, e.g., $\varepsilon=10^{-6}$, which guarantees that $f_{i, n, \ell} \neq f_{k, m, \lambda}$ and $f_{i, n-1, \ell}<f_{i, n, \ell}+\varepsilon<f_{i, n+1, \ell}$ hold. With the resulting new sets of discrete frequencies $\left\{f_{i, n, \ell}\right\}$, the performance degradation of the channel simulator can completely be neglected and the uncorrelatedness between different processes is confirmed.

By using (21), we can show that (11) approaches the desired Rayleigh distribution (2) if $N_{i, \ell} \rightarrow \infty$. As mentioned above, the condition (20) guarantees that (14), (15), and (17) are identical with (4), (5), and (7), respectively. It can also be shown that the substitution of (21) and (22) into (13), (16), and (18) results for $N_{i, \ell} \rightarrow \infty$ in $\tilde{r}_{\mu_{i, \ell} \mu_{i, \ell}}(\tau) \rightarrow r_{\mu_{i, \ell} \mu_{i, \ell}}(\tau)$, $\tilde{r}_{\mu_{\ell} \mu_{\ell}}(\tau) \rightarrow r_{\mu_{\ell} \mu_{\ell}}(\tau)$, and $\tilde{r}_{\zeta_{\ell}^{2} \zeta_{\ell}^{2}}(\tau) \rightarrow r_{\zeta_{\ell}^{2} \zeta_{\ell}^{2}}(\tau)$, respectively. Fig. 1 impressively illustrates the excellent agreement between the Rayleigh distribution in (1) with $\sigma_{0}^{2}=1$ and the approximate envelope PDF $\tilde{p}_{\zeta_{\ell}}(x)$ in (11) with $N_{1, \ell}=9$ and $N_{2, \ell}=$ 10. The corresponding simulated envelope PDF obtained from the output of the channel simulator is also presented in the figure to validate the analytical result. Fig. 2 shows the ACF $\tilde{r}_{\mu_{i, \ell} \mu_{i, \ell}}(\tau)$ with $N_{i, \ell}=10$ and the CCF $\tilde{r}_{\mu_{1, \ell} \mu_{2, \ell}}(\tau)$ with $N_{1, \ell}=9$ and $N_{2, \ell}=10$ by using the MEDS. Again, the simulation results are provided for reasons of verification. The ACF $r_{\mu_{i, \ell} \mu_{i, \ell}}(\tau)$ and CCF $r_{\mu_{1, \ell} \mu_{2, \ell}}(\tau)$ of the reference model are also demonstrated in the figure for comparison purposes. Clearly, $\tilde{r}_{\mu_{1, \ell} \mu_{2, \ell}}(\tau)=r_{\mu_{1, \ell} \mu_{2, \ell}}(\tau)=0$ holds for all $\tau$. The ACF $\tilde{r}_{\mu_{i, \ell} \mu_{i, \ell}}(\tau)$ matches almost perfectly the desired 


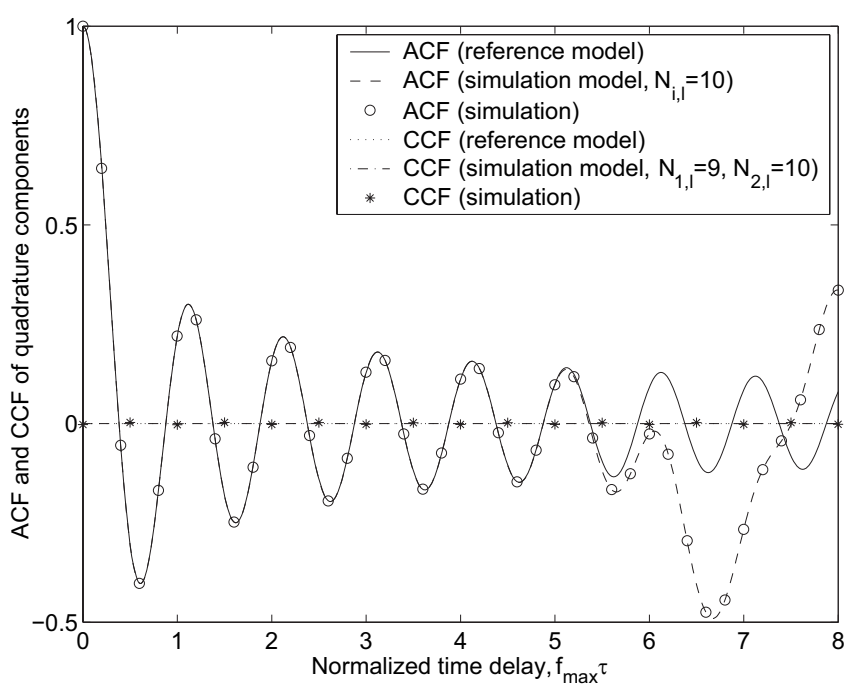

Fig. 2. The ACFs and CCFs of the quadrature components of the reference model and the simulation model by using the MEDS $\left(\sigma_{0}^{2}=1\right)$.

one $r_{\mu_{i, \ell} \mu_{i, \ell}}(\tau)$ if the normalized time delay $f_{\max } \tau$ is within the interval $\left[0, N_{i, \ell} / 2\right]$, which includes $N_{i, \ell}$ zero-crossings of $r_{\mu_{i, \ell} \mu_{i, \ell}}(\tau)$. In case that $f_{\max } \tau>N_{i, \ell} / 2, \tilde{r}_{\mu_{i, \ell} \mu_{i, \ell}}(\tau)$ and $r_{\mu_{i, \ell} \mu_{i, \ell}}(\tau)$ will diverge gradually and never converge again. Clearly, with the increase of $N_{i, \ell}$, a better approximation can be achieved over larger time delays. It is also shown in Fig. 3 that the ACFs of the complex envelope of the reference model and simulation model are very close to each other when $f_{\max } \tau$ is located in the interval $\left[0, \min \left\{N_{1, \ell}, N_{2, \ell}\right\} / 2\right]$. For $N_{1, \ell}=9$ and $N_{2, \ell}=10$, Fig. 4 indicates that the squared envelope ACF of the simulator gives a fairly good approximation to the desired one if $f_{\max } \tau$ is within the interval $\left[0, \min \left\{N_{1, \ell}, N_{2, \ell}\right\} / 2\right]$. It is interesting to observe that $\tilde{r}_{\zeta_{\ell}^{2} \zeta_{\ell}^{2}}(\tau)<r_{\zeta_{\ell}^{2} \zeta_{\ell}^{2}}(\tau)$ always holds within this interval. For example, at the origin $\tau=0, \tilde{r}_{\zeta_{\ell}^{2} \zeta_{\ell}^{2}}(0)=\sigma_{0}^{4}\left(8-\frac{3}{2 N_{1, \ell}}-\frac{3}{2 N_{2, \ell}}\right)$ is always smaller than $r_{\zeta_{\ell}^{2} \zeta_{\ell}^{2}}(0)=8 \sigma_{0}^{4}$ for finite $N_{1, \ell}$ and $N_{2, \ell}$. The simulation result obtained by using $N_{1, \ell}=159$ and $N_{2, \ell}=160$ clearly demonstrates that $\tilde{r}_{\zeta_{\ell}^{2} \zeta_{\ell}^{2}}(\tau)$ will tend to $r_{\zeta_{\ell}^{2} \zeta_{\ell}^{2}}(\tau)$ when both $N_{1, \ell}$ and $N_{2, \ell}$ are sufficiently large. Due to the fact that short time delays, e.g., $f_{\max } \tau \leq 0.3$, are of more interest for most communication systems [10], the MEDS with small numbers of sinusoids $N_{i, \ell}$ is indeed an excellent method in terms of the above interested correlation properties. In Fig. 5, we vividly present two uncorrelated simulated fading envelopes by using the MEDS with $\sigma_{0}^{2}=1, f_{\max }=91 \mathrm{~Hz}$, $N_{1,1}=9, N_{2,1}=10, N_{1,2}=8$, and $N_{2,2}=12$.

\section{B. $L P N M$}

By using the LPNM, $c_{i, n, \ell}$ and $\theta_{i, n, \ell}$ in (11) are the same as those given for the MEDS, while the discrete frequencies $f_{i, n, \ell}$ are determined by minimizing the following error norm

$$
\begin{aligned}
E_{\ell}= & \left\{\frac{W_{1}}{\tau_{\max _{1}}} \int_{0}^{\tau_{\max _{1}}}\left|r_{\mu_{1, \ell} \mu_{1, \ell}}(\tau)-\tilde{r}_{\mu_{1, \ell} \mu_{1, \ell}}(\tau)\right|^{p_{1}} d \tau\right\}^{1 / p_{1}} \\
& +\left\{\frac{W_{2}}{\tau_{\max _{2}}} \int_{0}^{\tau_{\max _{2}}}\left|r_{\mu_{2, \ell} \mu_{2, \ell}}(\tau)-\tilde{r}_{\mu_{2, \ell} \mu_{2, \ell}}(\tau)\right|^{p_{2}} d \tau\right\}^{1 / p_{2}}
\end{aligned}
$$

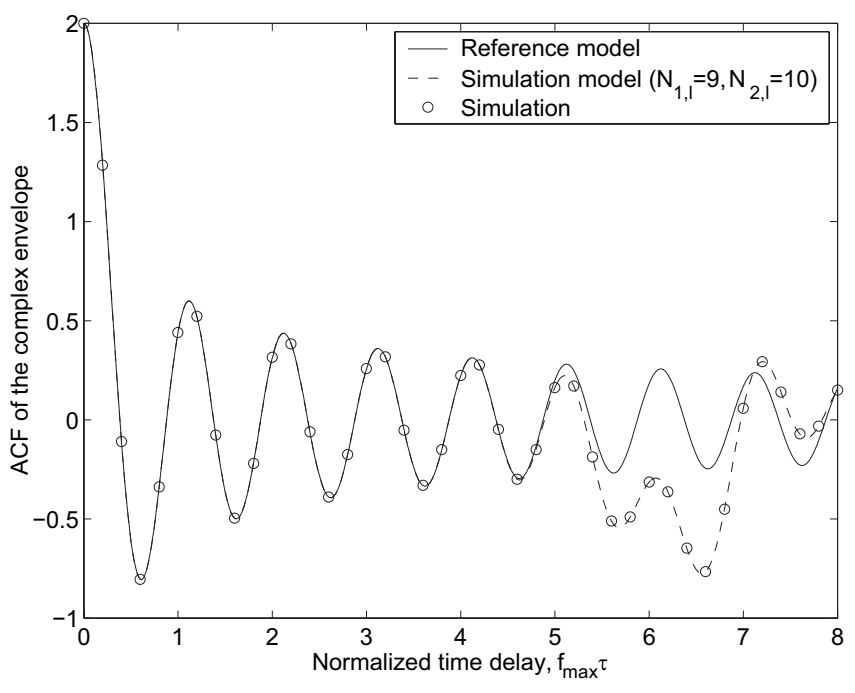

Fig. 3. The ACFs of the complex envelope of the reference model and the simulation model by using the $\operatorname{MEDS}\left(\sigma_{0}^{2}=1\right)$.

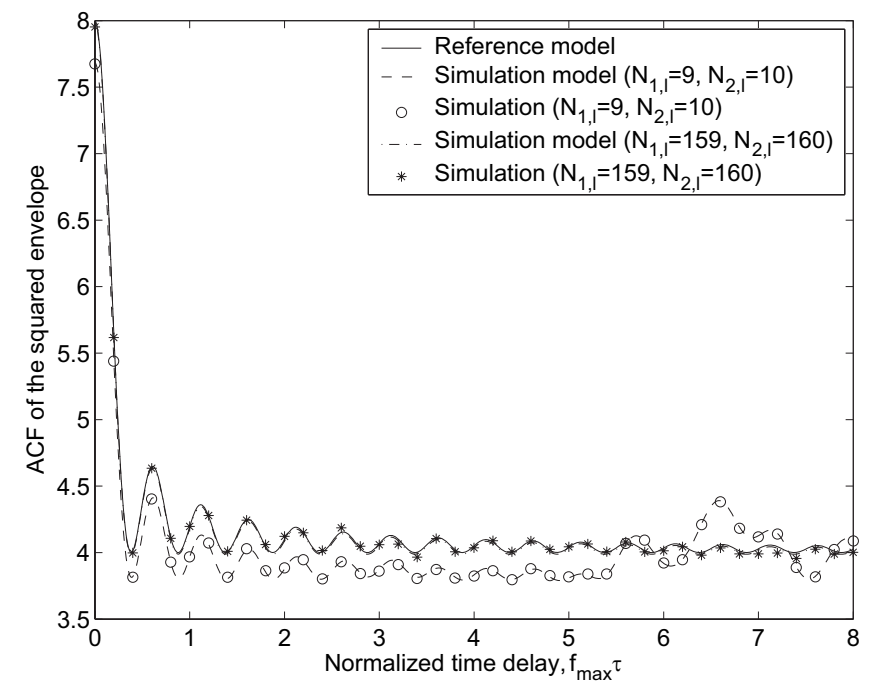

Fig. 4. The ACFs of the squared envelope of the reference model and the simulation model by using the MEDS $\left(\sigma_{0}^{2}=1\right)$.

$$
\begin{aligned}
& +\left\{\frac{W_{3}}{\tau_{\max _{2}}} \int_{0}^{\tau_{\max 2}}\left|r_{\zeta_{\ell}^{2} \zeta_{\ell}^{2}}(\tau)-\tilde{r}_{\zeta_{\ell}^{2} \zeta_{\ell}^{2}}(\tau)\right|^{p_{2}} d \tau\right\}^{1 / p_{2}}, \\
& p_{1}, p_{2}=1,2, \ldots
\end{aligned}
$$

where $\tau_{\max _{1}}=N_{1, \ell} /\left(2 f_{\max }\right)$ and $\tau_{\max _{2}}=N_{2, \ell} /\left(2 f_{\max }\right)$ define the maximum time delays up to which the approximation to the desired ACFs is of interest. The appropriate weighting functions $W_{1}, W_{2}$, and $W_{3}$ have to be chosen empirically, e.g., one can use the scaled versions of $r_{\mu_{1, \ell} \mu_{1, \ell}}(\tau)$, $r_{\mu_{2, \ell} \mu_{2, \ell}}(\tau)$, and $r_{\zeta_{\ell}^{2} \zeta_{\ell}^{2}}(\tau)$, respectively. The optimized sets of discrete frequencies $f_{1, n, \ell}$ and $f_{2, n, \ell}$ will be attained by applying a numerical optimization algorithm. It is important to mention that the global minimum of $E_{\ell}$ cannot be guaranteed to be found by any optimization algorithm. In general, a local minimum of $E_{\ell}$ is obtained. The advantage we may take from this property is that various local minima lead to various disjoint sets of discrete frequencies $f_{i, n, \ell}$. Therefore, we can easily satisfy the inequality (20) by taking one or 


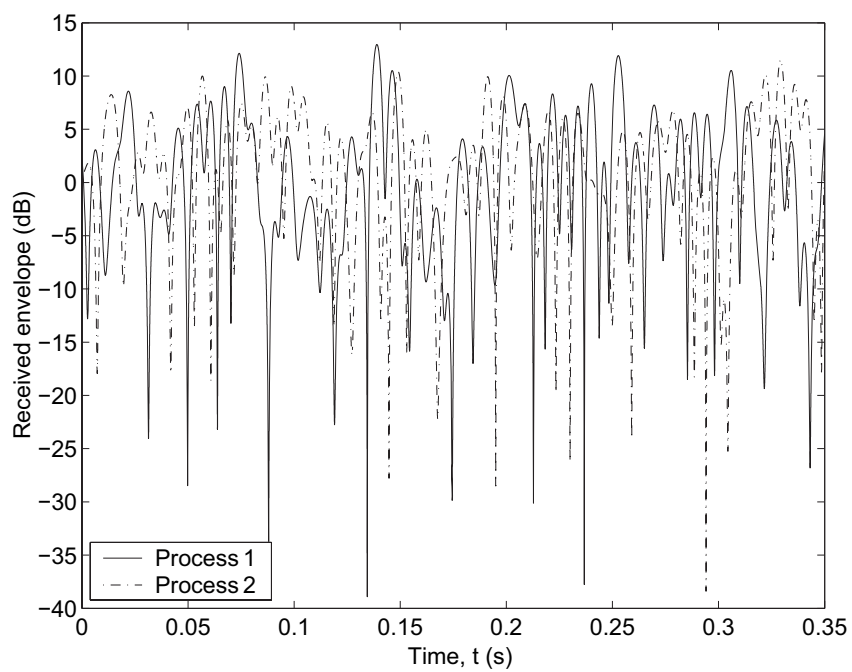

Fig. 5. Uncorrelated simulated fading envelopes by using the MEDS $\left(\sigma_{0}^{2}=\right.$ $\left.1, f_{\max }=91 \mathrm{~Hz}, N_{1,1}=9, N_{2,1}=10, N_{1,2}=8, N_{2,2}=12\right)$.

a combination of the following four measures: 1) choosing the numbers of sinusoids $N_{i, \ell}$ such that the relation (24) is fulfilled, 2) minimizing (25) by using different values of $p_{1}$ and $p_{2}, 3$ ) minimizing (25) with different values of $\tau_{\max _{1}}$ and $\tau_{\max _{2}}$, and 4) carrying out the optimization by using different starting values for $f_{i, n, \ell}$. Jakes' method [1] and the MEDS can, e.g., be employed here. It should be stressed that the LPNM controlled by any of the last three measures can be used to design $\mathcal{L}$ uncorrelated waveforms even when $N_{i, \ell}=N_{k, \lambda}$ holds for $i, k=1,2$ and $\ell, \lambda=1,2, \ldots, \mathcal{L}$.

Since the expression (21) applies to both the MEDS and the LPNM, the envelope PDF $\tilde{p}_{\zeta_{\ell}}(x)$ [see (11)] by using the LPNM is identical to the result shown in Fig. 1 with the same parameters. In Fig. 6, the optimized squared envelope ACF $\tilde{r}_{\zeta_{\ell}^{2} \zeta_{\ell}^{2}}(\tau)$ with $N_{1, \ell}=9$ and $N_{2, \ell}=10$ is compared with the desired one $r_{\zeta_{\ell}^{2}} \zeta_{\ell}^{2}(\tau)$ and the approximate one by using the MEDS. As starting values for the optimization of the discrete frequencies $f_{i, n, \ell}$, the expression (22) determined by the MEDS was used. Also, $p_{1}=p_{2}=2$ were selected. We observe that the approximate squared envelope ACF by using the LPNM nearly coincides with that by using the MEDS when $f_{\max } \tau$ is located in the interval $\left[0, \min \left\{N_{1, \ell}, N_{2, \ell}\right\} / 2\right]$. By using the same parameters, the optimized ACFs $\tilde{r}_{\mu_{i, \ell} \mu_{i, \ell}}(\tau)$ and $\tilde{r}_{\mu_{\ell} \mu_{\ell}}(\tau)$ also show quite similar results to those obtained by using the MEDS. For shortness, they are not shown here.

In short, both the MEDS and the LPNM can provide similar good approximations to the desired statistical properties of the reference model. In order to guarantee the uncorrelatedness between different simulated processes, the LPNM is more flexible than the MEDS, but one has to pay higher numerical computation expenditure. Due to the fact that the parameters $f_{i, n, \ell}$ determined by the LPNM are not available in a closedform expression, it is impossible to evaluate the convergency of the relevant correlation functions of the simulation model to those of the reference model when the numbers of sinusoids tend to infinity. Just the reverse holds for the MEDS.

Compared with the non-ergodic stochastic SoS channel simulators in [5], [6], the presented deterministic SoS channel simulator with the MEDS and LPNM has much better

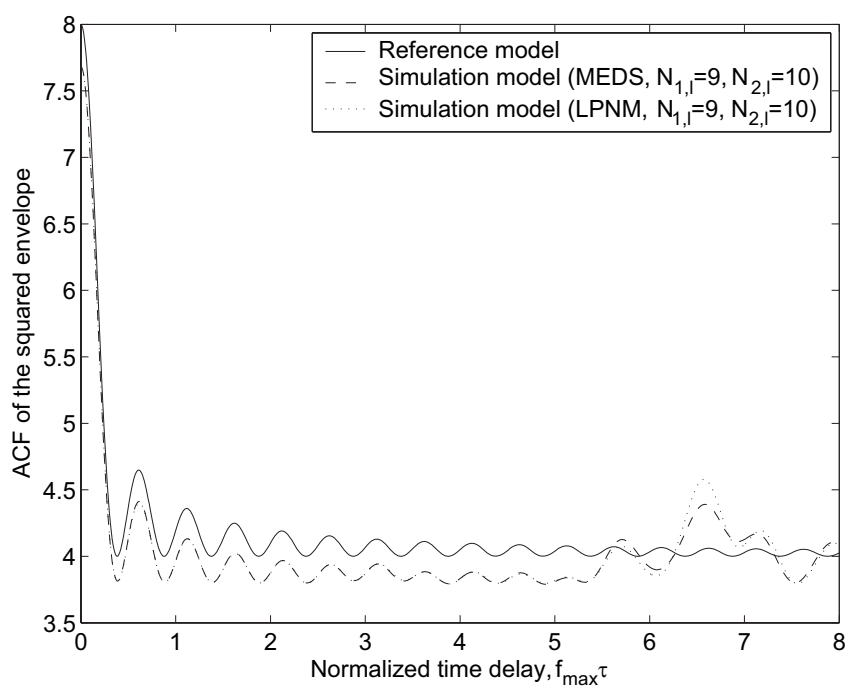

Fig. 6. The ACFs of the squared envelope of the reference model and the simulation model by using the MEDS and the LPNM $\left(\sigma_{0}^{2}=1\right)$.

simulation efficiency since the calculation of its statistical properties does not need the average of a number of random trials. With the same numbers of sinusoids, our deterministic channel simulator has similar performance to that of the stochastic simulators in [5], [6] in terms of the amplitude PDF. The performance of the deterministic channel simulator is comparable to or even better than that of the stochastic channel simulators in [5], [6] for the approximation of the ACFs inside the specified time delay ranges, e.g., $f_{\max } \tau \in\left[0, N_{i, \ell} / 2\right]$ for $r_{\mu_{i, \ell} \mu_{i, \ell}}(\tau)$. Outside the specified time delay ranges, which may not be relevant for communication systems [10], the stochastic channel simulators in [5], [6] provide much better approximations to the desired ACFs than our deterministic channel simulator. Furthermore, the accuracy of the statistic properties of the non-ergodic stochastic channel simulators can be improved by increasing either the numbers of sinusoids or the number of random trials to be averaged. On the other hand, the performance of the presented deterministic channel simulator can only be improved by increasing the numbers of sinusoids.

It is well known that multiple cross-correlated processes can be obtained by using a linear combination of uncorrelated processes [9], [11]. As shown in [11], the above presented deterministic channel simulator can easily be extended to the generation of multiple cross-correlated Rayleigh fading processes for simulating more realistic MIMO channels.

\section{CONCLUSION}

In this letter, two parameter computation methods for deterministic SoS channel simulators are presented to generate multiple uncorrelated Rayleigh fading processes, which are useful for the modeling of wideband, MIMO, and diversitycombined multipath fading channels. Compared with the MEDS, the LPNM has higher numerical computation expenditure. In order to guarantee the uncorrelatedness between different simulated fading processes, the LPNM is a more flexible method than the MEDS. In both cases, the statistical 
properties of the simulated processes match very closely the desired properties of the reference model.

\section{APPENDIX I}

\section{DERIVATION OF (18)}

In this appendix, we derive the time-averaged ACF $\tilde{r}_{\zeta_{\ell}^{2} \zeta_{\ell}^{2}}(\tau)$ of the squared envelope for the deterministic simulation model in (9). We have

$$
\begin{aligned}
\tilde{r}_{\zeta_{\ell}^{2} \zeta_{\ell}^{2}}(\tau)= & \lim _{T \rightarrow \infty} \frac{1}{2 T} \int_{-T}^{T} \tilde{\zeta}_{\ell}^{2}(t) \tilde{\zeta}_{\ell}^{2}(t+\tau) d t \\
= & \lim _{T \rightarrow \infty} \frac{1}{2 T} \int_{-T}^{T}\left[\tilde{\mu}_{1, \ell}^{2}(t)+\tilde{\mu}_{2, \ell}^{2}(t)\right] \\
& \cdot\left[\tilde{\mu}_{1, \ell}^{2}(t+\tau)+\tilde{\mu}_{2, \ell}^{2}(t+\tau)\right] d t \\
= & \tilde{r}_{\mu_{1, \ell}^{2} \mu_{1, \ell}^{2}}(\tau)+\tilde{r}_{\mu_{2, \ell}^{2} \mu_{2, \ell}^{2}}(\tau) \\
& +\tilde{r}_{\mu_{1, \ell}^{2} \mu_{2, \ell}^{2}}(\tau)+\tilde{r}_{\mu_{2, \ell}^{2} \mu_{1, \ell}^{2}}(\tau) .
\end{aligned}
$$

For the above equation, we first compute $\tilde{r}_{\mu_{1, \ell}^{2} \mu_{1, \ell}^{2}}(\tau)$. The remaining terms on the right hand side of (26) can be computed in a similar manner. Then,

$$
\begin{aligned}
\tilde{r}_{\mu_{1, \ell}^{2} \mu_{1, \ell}^{2}}(\tau)= & \lim _{T \rightarrow \infty} \frac{1}{2 T} \int_{-T}^{T} \tilde{\mu}_{1, \ell}^{2}(t) \tilde{\mu}_{1, \ell}^{2}(t+\tau) d t \\
= & \lim _{T \rightarrow \infty} \frac{1}{2 T} \int_{-T}^{T} \sum_{n=1}^{N_{1, \ell}} \sum_{m=1}^{N_{1, \ell}} \sum_{p=1}^{N_{1, \ell}} \sum_{q=1}^{N_{1, \ell}} c_{1, n, \ell} c_{1, m, \ell} \\
& \cdot c_{1, p, \ell} c_{1, q, \ell} \cos \left(2 \pi f_{1, n, \ell} t+\theta_{1, n, \ell}\right) \\
& \cdot \cos \left(2 \pi f_{1, m, \ell} t+\theta_{1, m, \ell}\right) \\
& \cdot \cos \left[2 \pi f_{1, p, \ell}(t+\tau)+\theta_{1, p, \ell}\right] \\
& \cdot \cos \left[2 \pi f_{1, q, \ell}(t+\tau)+\theta_{1, q, \ell}\right] d t
\end{aligned}
$$

In (27), all terms are zero except the following four terms with: 1) $n=m=p=q$; 2) $n=m, p=q, n \neq p$; 3) $n=p$, $m=q, n \neq m$; 4) $n=q, m=p, n \neq m$. Next, each of these four terms is calculated individually to derive the overall expression of (27).

For Term $1(n=m=p=q)$, we have

$$
\begin{aligned}
& \lim _{T \rightarrow \infty} \frac{1}{2 T} \int_{-T}^{T} \sum_{n=1}^{N_{1, \ell}} c_{1, n, \ell}^{4} \cos ^{2}\left(2 \pi f_{1, n, \ell} t+\theta_{1, n, \ell}\right) \\
\cdot & \cos ^{2}\left[2 \pi f_{1, n, \ell}(t+\tau)+\theta_{1, n, \ell}\right] d t \\
= & \lim _{T \rightarrow \infty} \frac{1}{2 T} \int_{-T}^{T} \sum_{n=1}^{N_{1, \ell}} \frac{c_{1, n, \ell}^{4}}{4}\left[1+\cos \left(4 \pi f_{1, n, \ell} t+2 \theta_{1, n, \ell}\right)\right] \\
& \cdot\left\{1+\cos \left[4 \pi f_{1, n, \ell}(t+\tau)+2 \theta_{1, n, \ell}\right]\right\} d t \\
= & \sum_{n=1}^{N_{1, \ell}} \frac{c_{1, n, \ell}^{4}}{4}\left[1+\frac{1}{2} \cos \left(4 \pi f_{1, n, \ell} \tau\right)\right] .
\end{aligned}
$$

For Term $2(n=m, p=q, n \neq p)$, we have

$$
\begin{aligned}
& \lim _{T \rightarrow \infty} \frac{1}{2 T} \int_{-T}^{T} \sum_{n=1}^{N_{1, \ell}} c_{1, n, \ell}^{2} \cos ^{2}\left(2 \pi f_{1, n, \ell} t+\theta_{1, n, \ell}\right) \\
& \cdot \sum_{p=1, p \neq n}^{N_{1, \ell}} c_{1, p, \ell}^{2} \cos ^{2}\left[2 \pi f_{1, p, \ell}(t+\tau)+\theta_{1, p, \ell}\right] d t \\
= & \sum_{n=1}^{N_{1, \ell}} \frac{c_{1, n, \ell}^{2}}{2} \sum_{p=1, p \neq n}^{N_{1, \ell}} \frac{c_{1, p, \ell}^{2}}{2} \\
= & \left(\sum_{n=1}^{N_{1, \ell}} \frac{c_{1, n, \ell}^{2}}{2}\right)^{2}-\sum_{n=1}^{N_{1, \ell}} \frac{c_{1, n, \ell}^{4}}{4} .
\end{aligned}
$$

For Term $3(n=p, m=q, n \neq m)$, we have

$$
\begin{aligned}
& \lim _{T \rightarrow \infty} \frac{1}{2 T} \int_{-T}^{T} \sum_{n=1}^{N_{1, \ell}} c_{1, n, \ell}^{2} \cos \left(2 \pi f_{1, n, \ell} t+\theta_{1, n, \ell}\right) \\
\cdot & \cos \left[2 \pi f_{1, n, \ell}(t+\tau)+\theta_{1, n, \ell}\right] \\
& \cdot \sum_{m=1, m \neq n}^{N_{1, \ell}} c_{1, m, \ell}^{2} \cos \left(2 \pi f_{1, m, \ell} t+\theta_{1, m, \ell}\right) \\
& \cdot \cos \left[2 \pi f_{1, m, \ell}(t+\tau)+\theta_{1, m, \ell}\right] d t \\
= & \sum_{n=1}^{N_{1, \ell}} \frac{c_{1, n, \ell}^{2}}{2} \cos \left(2 \pi f_{1, n, \ell} \tau\right) \\
& \cdot \sum_{n=1, m \neq n}^{N_{1, \ell}} \frac{c_{1, m, \ell}^{2}}{2} \cos \left(2 \pi f_{1, m, \ell} \tau\right) \\
= & {\left[\sum_{n=1}^{N_{1, \ell}} \frac{c_{1, n, \ell}^{2}}{2} \cos \left(2 \pi f_{1, n, \ell} \tau\right)\right]^{2} } \\
& \cdot-\sum_{n=1}^{N_{1, \ell}} \frac{c_{1, n, \ell}^{4}}{4} \cos ^{2}\left(2 \pi f_{1, n, \ell} \tau\right) .
\end{aligned}
$$

It can be shown that Term $4(n=q, m=p, n \neq m)$ is equal to Term 3 . Hence,

$$
\begin{aligned}
\tilde{r}_{\mu_{1, \ell}^{2} \mu_{1, \ell}^{2}}(\tau)= & \left(\sum_{n=1}^{N_{1, \ell}} \frac{c_{1, n, \ell}^{2}}{2}\right)^{2} \\
+ & 2\left[\sum_{n=1}^{N_{1, \ell}} \frac{c_{1, n, \ell}^{2}}{2} \cos \left(2 \pi f_{1, n, \ell} \tau\right)\right]^{2} \\
& -\sum_{n=1}^{N_{1, \ell}} \frac{c_{1, n, \ell}^{4}}{8}\left[2+\cos \left(4 \pi f_{1, n, \ell} \tau\right)\right] .
\end{aligned}
$$

By analogy, we can show that

$$
\begin{aligned}
\tilde{r}_{\mu_{2, \ell}^{2} \mu_{2, \ell}^{2}}(\tau)= & \left(\sum_{n=1}^{N_{2, \ell}} \frac{c_{2, n, \ell}^{2}}{2}\right)^{2} \\
& +2\left[\sum_{n=1}^{N_{2, \ell}} \frac{c_{2, n, \ell}^{2}}{2} \cos \left(2 \pi f_{2, n, \ell} \tau\right)\right]^{2}
\end{aligned}
$$




$$
\begin{aligned}
& -\sum_{n=1}^{N_{2, \ell}} \frac{c_{2, n, \ell}^{4}}{8}\left[2+\cos \left(4 \pi f_{2, n, \ell} \tau\right)\right] \\
\tilde{r}_{\mu_{1, \ell}^{2} \mu_{2, \ell}^{2}}(\tau)= & \sum_{n=1}^{N_{1, \ell}} \frac{c_{1, n, \ell}^{2}}{2} \sum_{m=1}^{N_{2, \ell}} \frac{c_{2, m, \ell}^{2}}{2} \\
\tilde{r}_{\mu_{2, \ell}^{2} \mu_{1, \ell}^{2}}(\tau)= & \sum_{n=1}^{N_{1, \ell}} \frac{c_{1, n, \ell}^{2}}{2} \sum_{m=1}^{N_{2, \ell}} \frac{c_{2, m, \ell}^{2}}{2} .
\end{aligned}
$$

The substitution of (31)-(34) into (26) gives the final result of $\tilde{r}_{\zeta_{\ell}^{2} \zeta_{\ell}^{2}}(\tau)$, as shown in (18).

\section{REFERENCES}

[1] W. C. Jakes, Ed., Microwave Mobile Communications. New York: WileyIEEE Press, 1994.

[2] P. Dent, G. E. Bottomley, and T. Croft, "Jakes fading model revisited," Electron. Lett., vol. 29, no. 13, pp. 1162-1163, June 1993.

[3] Y. B. Li and Y. L. Guan, "Modified Jakes model for simulating multiple uncorrelated fading waveforms," in Proc. IEEE ICC'00, pp. 46-49.
[4] Y. X. Li and X. J. Huang, "The simulation of independent Rayleigh faders," IEEE Trans. Commun., vol. 50, no. 9, pp. 1503-1514, Sept. 2002.

[5] Y. R. Zheng and C. S. Xiao, "Improved models for the generation of multiple uncorrelated Rayleigh fading waveforms," IEEE Commun. Lett., vol. 6, no. 6, pp. 256-258, June 2002.

[6] Y. R. Zheng and C. S. Xiao, "Simulation models with correct statistical properties for Rayleigh fading channels," IEEE Trans. Commun., vol. 51, no. 6, pp. 920-928, June 2003.

[7] M. Pätzold, Mobile Fading Channels. Chichester: John Wiley \& Sons, 2002.

[8] R. H. Clarke, "A statistical theory of mobile-radio reception," Bell Syst. Tech. J., vol. 47, pp. 957-1000, July/Aug. 1968.

[9] G. L. Stüber, Principles of Mobile Communications, Second Edition. Boston: Kluwer Academic Publishers, 2001.

[10] P. Hoeher and A. Steingass, "Modeling and emulation of multipath fading channels using controlled randomness," in Proc. ITG-Fachtagung Wellenausbreitung bei Funksystemen und Mikrowellensystemen, 1998, pp. 209-220.

[11] C. X. Wang and M. Pätzold, "Efficient simulation of multiple crosscorrelated Rayleigh fading channels," in Proc. IEEE PIMRC'03, pp. 1526-1530. 